\title{
Electron holography - where we are and where to go
}

\author{
Hannes Lichte
}

Institute of Applied Physics, Dresden University, D 01062 Dresden, Germany, www.triebenberg.de

\section{Visions:}

The ultimate goal of the different TEM-methods is to answer the most essential questions in solidstate physics and materials science, such as Which atom is where? Which magnetic or electric fields are around? Which is the binding structure? However, the restricted transfer properties of conventional TEM imaging prevent one from reaching this goal: shortcomings are immanent, such as nonlinearity, phase loss in the image intensity, irretrievable cross talk between amplitude and phase of the object exit wave in the image intensity, delocalisation of seemingly resolved structures, and mixing of elastic and inelastic information. Furthermore, the relation between real space and reciprocal space is not sufficiently unique, wave optical tools for analysis of the results are poor, and the Stobbs-factor problem hampers a reliable interpretation by means of simulation calculations.

\section{Off-axis holography offers solutions:}

Holography is strictly linear and pure zero-loss $\left(10^{-15} \mathrm{eV}\right)$-imaging; the successful recording of inelastic holograms may shed new light on this interesting point. Furthermore, it provides amplitude and phase separately; one can correct all the coherent aberrations, also the ones to come up with improving resolution; there is an unique relation between real and reciprocal space, since everything is reconstructed from one hologram; the reconstructed wave can be exploited by means of all thinkable wave optical tools like nanodiffraction and analysis of diffracted waves; all data are quantitative, and, according to our latest findings, probably due to the inherent zero-loss-imaging, the Stobbs-factor is of minor importance. Holography seems the ideal method for reaching the goals, however, it is applicable to real-world problems only after developing the general performance.

\section{Optimum Performance:}

Holographic imaging requires specific parameters for optimum performance as described by resolution, field of view and noise properties. The biprism has to be installed at an optimum position in the TEM. Furthermore, there is an Optimum Focus optimising the gradient of the wave aberration in the TEM to give both maximum information limit and smallest possible point spread function, as well as minimal artefacts; also at the Optimum Focus, one achieves optimum sampling of the hologram by the CCD-camera both in real space and in Fourier space. Correction of aberrations needs a high accuracy of at least 10 aberration parameters. The noise level in the reconstructed wave determines the detection limit for weak objects; it is given by the contrast of the hologram fringes transferred to the computer and by the collected dose; besides a high brightness gun, one needs careful sampling by the CCD-camera and high stability of the whole microscope system including the environment; an optimum specimen thickness of twice the inelastic mean free path - whenever affordable e.g. at medium resolution - yields optimum signal/ noise ratio.

In summary, today's limits of holographic performance are given by brightness and stability, point spread function, pixel number, and accuracy of aberrations. 


\section{Problems of Applications}

After many years of development, electron holography has reached such a performance that, besides showing the "in-principle"-facilities, it has surmounted the state of basic development of the method and is applied to the solution of increasingly sophisticated real-world problems in materials science.

Medium Resolution:

For structure components larger than about 5 times the Scherzer resolution, aberrations can be neglected; for finer details one has to be very careful about delocalisation effects. With this in mind, the reconstructed phase images can be interpreted as the object phase structure, which is not at all discernible by conventional TEM methods. In a wide variety, magnetic microstructures are analysed in the range down to about 50nm. Trying to analyse finer magnetic structures, one finds that the reachable lateral resolution is limited by the strength of the phase shift: since the phase shift is given by the enclosed magnetic flux, it shrinks down with the size of the object details. For example, the magnetic phase shift due to a single Bohr Magneton in a unit cell can be estimated as $2 \pi 10^{-5}$, which, alas, is far beyond the phase detection limit of about $2 \pi / 30$ found at atomic resolution. The situation is much more advantageous with the electric phase shift integrating the electric potential locally along the electron trajectory. This is true for the electric fields measured e.g. in semiconductors, for dopant profiling, and for the analysis of polarisation in ferroelectrics. Optimising the object thickness, the phase shift can be evaluated well above noise also in the nanometer range, even down to atomic resolution.

\section{High Resolution:}

At high resolution, aberrations have to be corrected prior to any meaningful evaluation of the reconstructed wave. Correction of aberrations re-sorts the cross talk between amplitude and phase and heals delocalisation; it opens the effective objective aperture, consequently, it not only achieves improvement of resolution, additionally it enhances the measurable phase shift e.g. due to single atoms. Since the phase shift correlates with the atomic number, the phase image allows distinguishing between different atom species. As the Stobbs-factor seems only a minor problem with holography, the evaluation of the phase data by comparison with simulated ones opens a way for holographic materials analysis at an atomic scale.

\section{Outlook:}

After the first experiments by Moellenstedt and Wahl showing the principle of off-axis electron holography in 1968, the different holography groups around the world have developed electron holography as a meanwhile indispensable tool in the field of nanoanalysis. Much has been reached; still much is left to be done, for example:

- The fields in the specimen leak out producing 3D-fields, which influence both object wave and reference wave. In any case, the phase represents the whole 3D field, not only the inner object.

- The phase shift stems from many different contributions, e.g. magnetics, mean inner potential, semiconductor potentials, inner charges at interfaces, surface charges probably induced by the electron beam, also from thickness variations, and from dynamic interaction. For unique interpretation, these have to be sorted out, possibly by in-situ experiments.

- With the reconstruction of the object exit wave, we have not yet reached the final goal: the step back from the wave to the underlying object structure is a difficult Inverse Problem. 